\title{
Inflammatory Polyneuropathy after Bariatric Surgery: Report of Two Cases
}

\author{
Joana Carvalho Dias, Cecília de Medeiros Vidal and Marcos R.G. de Freitas*
}

Neurological Department, Peripheral Nerve Service, Antônio Pedro Hospital, Federal Fluminense University, Niterói RJ, Brazil

*Corresponding author: Marcos RG de Freitas, Neurological Department, Peripheral Nerve Service, Antônio Pedro Hospital, Federal Fluminense University, Niterói RJ, Brazil, Tel: +55 (21) 2629-5227; Fax: +55 (21) 2629-5228; E-mail: mgdefreitas@outlook.com

Rec date: Mar 20, 2016; Acc date: July 12, 2016; Pub date: July 14, 2016

Copyright: (c) 2016 Dias JC. This is an open-access article distributed under the terms of the Creative Commons Attribution License, which permits unrestricted use, distribution, and reproduction in any medium, provided the original author and source are credited.

\section{Abstract}

Obesity is an important health problem worldwide. The bariatric surgeries (BS) are becoming more frequent due to the high prevalence of obesity. Acute and chronic neurological complications have been reported after this procedure, and can result mainly from nutritional deficiency. There are some reports of peripheral neuropathies due to mechanical or inflammatory mechanisms.
\end{abstract}

Objective: To report two cases of inflammatory polyneuropathy (PN) after bariatric surgery, without nutritional deficiency.

Case reports: The first patient was a young woman who presents a painful sensorial-motor PN, months after a BS. The other patient was an old man with a pure painful small-fiber PN, one year after the BS. In both patients, there was no nutritional deficiency and blood "screening" for polyneuropathy was unremarkable. The patients underwent a sural nerve biopsy.

Results: The nerve biopsy demonstrated a mild inflammatory infiltrate around epineural vessels and a few axonal and demyelination changes. They were treated with intravenous immunoglobulin with good results.

Conclusion: Although most of the peripheral neuropathies complications after BS are due to nutritional deficiencies, some neuropathies are due to inflammatory involvement of the peripheral nerve. An autoimmune process has been accepted as the underlying pathophysiology in these cases. We think that the nerve biopsy is necessary to support this diagnosis in order to initiate an efficacious treatment.

Keywords: Bariatric surgery; Inflammatory infiltrates; Polyneuropathies

\section{Introduction}

Obesity is a chronic disease with high prevalence. The National Institutes of Health defines persons with obesity having a body mass index (BMI) above 30, and others with morbid obesity having BMI of 40 or more [1]. As an important global disease, it can lead to premature death and much comorbidity [1-5]. Complications of obesity include hypertension, diabetes mellitus type 2, sleep apnoea, idiopathic intracranial hypertension, arthralgia and hyperlipidaemia [2,6-8].

The epidemic of obesity and limited efficacy of available medical treatments follow by difficult and fail capacity to lose weight with appropriate diet and exercise combination have resulted in increasing utilization of bariatric surgery (BS) $[1,3,9,10]$. While BS is considered safe and effective, decreasing the comorbidities and improving the quality of life, it presents a number of risks [11-14].

In some cases BS has been accompanied by neurologic complications that may be irreversible [6-10]. Manifestations include encephalopathy, optic neuropathy, myelopathy, radiculoplexopathy, polyneuropathy $(\mathrm{PN})$ and mononeuropathy $[9,10]$. Neurological complications, mainly peripheral neuropathies, may occur after this procedure through mechanical, nutritional and inflammatory mechanisms [15]. The nutritional deficiency is the main cause of the peripheral neuropathies, although there are some reports of inflammatory causes [3,9-16]. Our objective is to report two patients with inflammatory PN after BS, which has been improved with intravenous immunoglobulin (IVIg).

\section{Cases Reports}

Case 1: A 35-year-old woman underwent BS in August 2013 (gastric bypass Roux-en-Y). Two months after surgery, with a weight loss of 25 $\mathrm{kg}$, the patient developed weakness in the lower extremities followed by pain and tingling in feet and hands, with difficulty walking. Neurologic examination revealed wasting and weakness in distal lower limbs (MRC grade 2 for foot and toes dorsiflexors with bilateral steppage gait, abolished tendon reflexes, superficial and vibratory hypoesthesia in distal lower limbs. The cranial nerves were normal. Laboratory tests were normal. Electroneuromyography (ENMG) showed a symmetrical axonal neuropathy. She was treated with multiple B vitamins orally for three months without improvement. Then a sural nerve biopsy was performed, showing a small collection of perivascular inflammatory mononuclear cells and mild mixed axonal and demyelinating features. (Figures $1 \mathrm{~A}$ and $1 \mathrm{~B}$ ). Soon after, she received a course of IVIg, with clinical improvement followed by IVIg 
Citation: Dias JC, Vidal CM, Freitas MRG (2016) Inflammatory Polyneuropathy after Bariatric Surgery: Report of Two Cases. J Neurol Disord 4:

Page 2 of 4

every month. Treatment was suspended after 20 months and she remains asymptomatic.
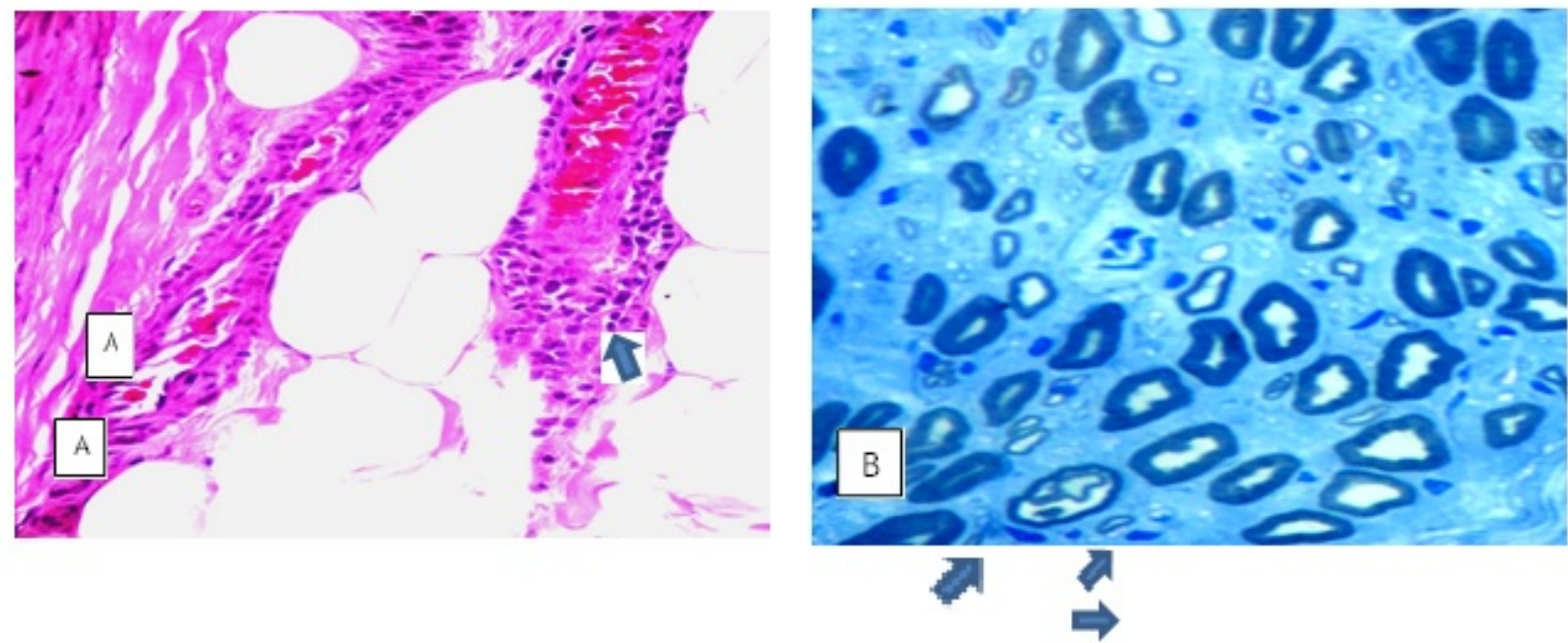

Figure 1: Case 1, A - Lymphocytic inflammatory infiltrate in the epineurium (arrow) H\&E 100X; B- Loss of myelinated fibers of large calibre, some fibers in remyelination (arrow) - Toluidine blue 400X.

Case 2: A 64 year-old-man, with morbid obesity refractory to medical treatment, with arterial hypertension, underwent bariatric surgery in May 2009 (gastric bypass Roux-en-Y) without complications. One year after he was submitted to a total thyroidectomy due a papillary carcinoma and initiated treatment with thyroid hormone. Before thyroidectomy, he had weight loss of $50 \mathrm{~kg}$ and developed numbness, tingling, and pain in distal lower and upper limbs. The neurologic examination showed normal strength and reflexes, however, there was evidence of diminished pinprick and thermal sensitivity distally in the lower limbs with associated allodynia. The ENMG was normal. He was treated with multiple vitamins, keeping levels within the normal range. As there was no improvement a sural nerve biopsy was performed, that showed a mild inflammatory infiltrate and some fibres with axonal and demyelination changes (Figures $2 \mathrm{~A}$ and $2 \mathrm{~B}$ ). Significant improvement was obtained with a course of IVIg for five days followed by IVIg every 20 days. Nowadays he still needs a course of IVIg every two months.
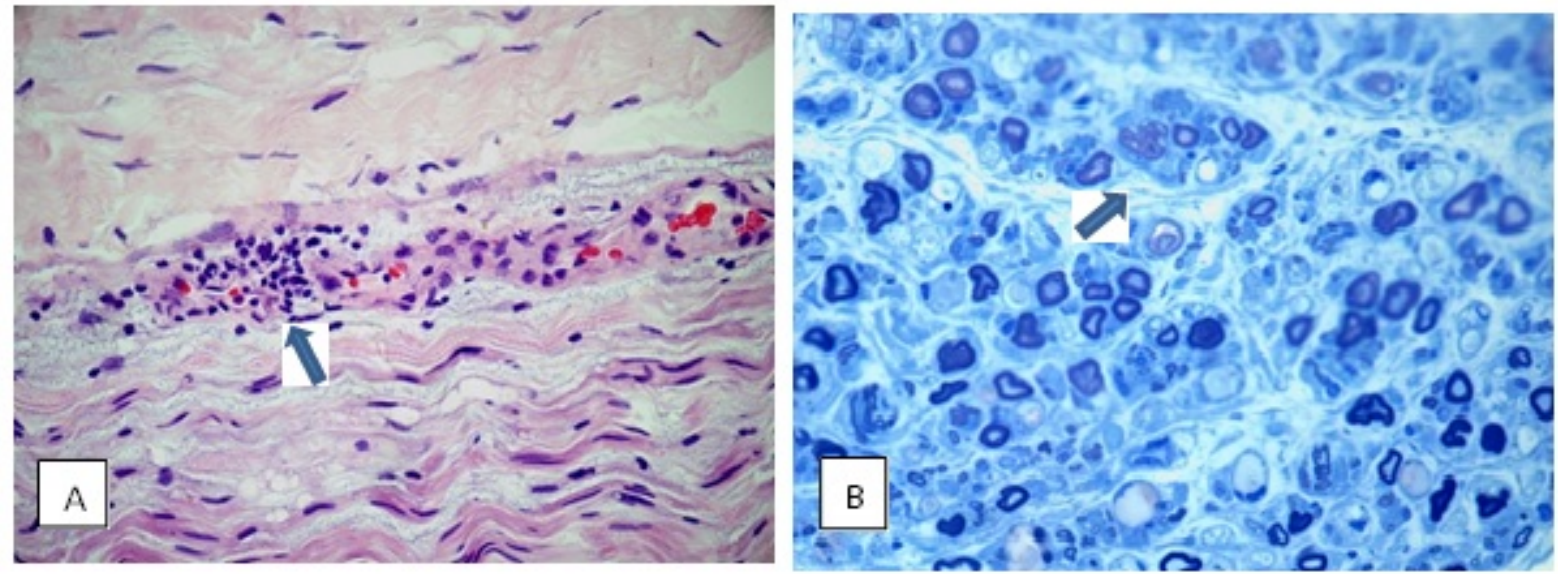

Figure 2: Case 2, A- Lymphocytic inflammatory infiltrate in endoneurium (arrow) H\&E 100X; B- Loss of large caliber fibers, remyelination in fibers (arrow) -Toluidine blue 100X.

\section{Discussion}

One of the most effective treatments for obesity and its associated comorbidities is BS because of its dramatic and durable results.
Usually, the patients have rapid and sustained weight loss [17]. The weight loss, especially when it occurs quickly, is the most well defined risk factor [15]. The gastric bypass Roux-en-Y is the most performed 
Page 3 of 4

procedure, and has proven to be the safest and most effective one $[7,17]$.

Complications related to the central or peripheral nervous system or both may be seen in approximately $5 \%$ to $16 \%$ of patients $[9,10,13]$.

Neurologic complications after BS are defined with an acute onset, when starts up to a month after surgery; sub-acute onset, when starts between one and three months after surgery or chronic onset when it starts more than three months after surgery $[3,16]$. It can involve any level of neuraxis. Its includes encephalopathy, optic neuropathy, myelopathy, myeloneuropathy, radiculoplexopathy, $\mathrm{PN}$ and mononeuropathy, resulting in carpal tunnel syndrome, meralgia paresthetica or footdrop $[9,10,13,16]$.

PN usually have a chronic evolution, but can evolve of an acute or sub-acute manner. Symptoms involve distal, painful paresthesias ("burning feet syndrome") and loss of pinprick and temperature sensation $[3,10]$. Neurological deficits are most commonly associated with nutritional deficiencies that develop after surgery. There are many factors implicated in malabsorption of nutrients: 1- decreased gastric acidity; 2-bypassed proximal intestines resulting in limited absorption due to shorter food contact time; 3-increased frequency of bowel movements and diarrhea associated with dumping syndrome and blind loop syndrome; 4- functional exocrine insufficiency $[10,18]$. Commonly implicated nutrient deficiencies in patients with a history of BS and neurologic complications include vitamin B12, thiamine, folate, vitamin D, vitamin E and copper deficiencies [10,19]. Multiple nutrient deficiencies may coexist. In some cases, a specific nutrient deficiency is not identified. The peripheral neuropathies best described are an acute, frequently painful $\mathrm{PN}$ or polyradiculoneuropathy associated with thiamine deficiency, and an isolated PN or myeloneuropathy associated with deficiencies of either vitamin B12 or copper [1,9].

Polyradiculoneuropathy, often mimicking Guillain-Barré syndrome (GBS) has been associated with vitamin B1 deficiency and has been seen as early as six weeks after surgery [9] These patients usually have axonal degeneration and a normal cerebrospinal fluid protein level $[9,10,17]$. Symptoms are often described as pain in the feet or low back with ascending paresthesias and may progress to leg weakness associated with ataxia, abolished reflexes with proprioceptive sensory loss $[10,20]$. An autoimmunity process has been postulated as pathophysiology $[17,21]$.

Mononeuropathy may occur after BS [9]. Weight loss may predispose to compression mononeuropathies like carpal tunnel syndrome, peroneal neuropathy and less usually ulnar, radial and lateral femoral cutaneous neuropathies $[6,9,10]$.

Our two cases manifested clinically by a symmetrical PN. In both patients, there was no nutritional deficiency and the blood "screening" (Vitamin B12, B1 and B6 levels, folic acid, serum copper, fasting glucose, hemoglobin A1c, serum protein electrophoresis with immunofixation, thyroid-stimulating hormone, thyroxine, rheumatoid factor, homocysteine, methylmalonic acid, antinuclear antibodies, human immunodeficiency virus (HIV), Human T-cell lymphotropic virus 1 and 2 antibodies (HTLV 1 and 2), viral panel for hepatitis virus $\mathrm{B}$ and $\mathrm{C}$ ) was unremarkable. Hereditary PN were excluded as there was no family history. One of the cases had sub-acute onset with sensorymotor manifestation with significant pain component and evolution of weakness in the lower limbs. The other case had chronic onset (one year after BS) with a predominance of nociceptive symptoms characterized by a small fibre PN without autonomic disturbance. The
ENMG in the first patient shows axonal degeneration (pattern similar to that described in the literature) $[3,22]$ and in the second case, it was normal. The sural nerve biopsy showed in both cases lymphocytic inflammatory infiltrate in epineurium, with a microvasculitis pattern. There was also loss of fibres with demyelination and remyelination features.

Some authors have noted inflammatory changes in nerve biopsies from patients with PN after BS [11]. Kumar, Thaisetthawatkul et al. suggested a possible immune mediated or inflammatory basis $[9,16]$.

In the case-control series published by Mayo Clinic, the authors reported the results of five sural biopsies taken from patients with PN or poliradiculoneuropathy. They found in nerve biopsies varying degrees of inflammatory infiltrates $[11,16]$. Some authors think that an inflammatory process may be secondary to malnutrition and cachexia induced by BS $[11,16]$. However, these patients do not respond to nutritional therapy, like our cases.

As our patients had improvement with IVIg, we suggest an immunopathogenesis mechanism in these PN. We believe that although the nutritional deficiency is the most common cause of PN after BS, in some cases the role of inflammatory and immune mechanisms deserves further consideration, mainly when there were no improvement with nutritional and vitamin supplementation.

\section{References}

1. Rudnicki SA (2010) Prevention and treatment of peripheral neuropathy after bariatric surgery. Curr Treat Options Neurol 12: 29-36.

2. Murara JR, Macedo LLB, Liberali R (2008) Análise da Eficácia da Cirurgia Bariátrica na redução de peso corporal e no combate à obesidade mórbita. Rev Brasil Obes Nutr Emagrec 2: 87-99.

3. Menezes MS, Harada KO, Alvarez G (2008) Painful peripheral polyneuropathy after bariatric surgery. Case reports. Rev Bras Anestesiol 58: 252-259.

4. Melo IT, São Pedro M (2012) Dor musculoesquelética em membros inferiores de pacientes obesos antes e depois da cirurgia bariátrica. $\mathrm{ABCD}$ Arq Bras Cir Dig 25: 29-32.

5. Kromas ML, Mousa OY, John S (2015) Hyperammonemia-induced encephalopathy: A rare devastating complication of bariatric surgery. World J Hepatol 7: 1007-1011.

6. Koffman BM, Greenfield LJ, Ali II, Pirzada NA (2006) Neurologic complications after surgery for obesity. Muscle Nerve 33: 166-176.

7. Torezan EFG (2013) Revisão das principais deficiências de micronutriente no pós-operatório do Bypass Gástrico em Y de Roux. Int J Nutrol 6: 37-42.

8. Berger JR (2004) The neurological complications of bariatric surgery. Arch Neurol 61: 1185-1189.

9. Kumar N (2014) Neurologic complications of bariatric surgery. Continuum (Minneap Minn) 20: 580-597.

10. Becker DA, Balcer LJ, Galetta SL (2012) The neurological complications of nutritional deficiency following bariatric surgery.

11. Frantz DJ (2012) Neurologic complications of bariatric surgery: Involvement of central, peripheral, and enteric nervous systems. Cir Gastroenterol Reports 14: 367-372.

12. Moura-Grec PG, Assis VH, Cannabrava VP, Vieira VM, Siqueira TL, et al. (2012) Systemic consequences of bariatric surgery and its repercussions on oral health. Arq Bras Cir Dig 25: 173-177.

13. Fragoso YD, Alves-Leon SV, Anacleto Ade C, Brooks JB, Gama PD, et al. (2012) Neurological complications following bariatric surgery. Arq Neuropsiquiatr 70: 700-703.

14. Carvalho IR, Loscalzo IT, Freitas MF, Jordão RE, Friano Tde C (2012) Incidence of vitamin B12 deficiency in patients submitted to Fobi-Capella Roux-en-Y bariatric surgery. Arq Bras Cir Dig 25: 36-40. 
Citation: Dias JC, Vidal CM, Freitas MRG (2016) Inflammatory Polyneuropathy after Bariatric Surgery: Report of Two Cases. J Neurol Disord 4:

Page 4 of 4

15. Alves LF, Gonçalves RM, Cordeiro GV, Lauria MW, Ramos AV (2006) Beriberi after bariatric surgery: not an unusual complication. Report of two cases and literature review. Arq Bras Endocrinol Metabol 50: 564-568.

16. Thaisetthawatkul P, Collazo-Clavell ML, Sarr MG, Norell JE, Dyck PJ (2004) A controlled study of peripheral neuropathy after bariatric surgery. Neurology 63: 1462-1470.

17. Juhasz-Pocsine K, Rudnicki SA, Archer RL, Harik SI (2007) Neurologic complications of gastric bypass surgery for morbid obesity. Neurology 68 : 1843-1850.

18. Bordalo LA, Mourão DM, Bressan J (2011) Nutritional deficiencies after bariatric surgery: why they happen?. Acta Med Port 24 Suppl 4 1021-1028.
19. Landais A (2014) Neurological complications of bariatric surgery. Obes Surg 24: 1800-1807.

20. Landais AF (2014) Rare neurologic complication of bariatric surgery: acute motor axonal neuropathy (AMAN), a severe motor axonal form of the Guillain Barré syndrome. Surg Obes Relat Dis 10: e85-87.

21. Ishaque N, Khealani BA, Shariff AH, Wasay M (2015) Guillain-Barré syndrome (demyelinating) six weeks after bariatric surgery: A case report and literature review. Obes Res Clin Pract 9: 416-419.

22. Elias WJ, Pouratian N, Oskouian RJ, Schirmer B, Burns T (2006) Peroneal neuropathy following successful bariatric surgery. Case report and review of the literature. J Neurosurg 105: 631-635. 